Reprod. Nutr. Dévelop., 1986, 26 (6), 1265-1272.

\title{
Influence d'une supplémentation en acides aminés dans un régime à base de protéines de crabe chez des homards juvéniles (Homarus americanus)
}

\author{
G. GAGNÉ, A. D. BOGHEN, J. D. CASTELL $\left({ }^{*}\right)$
}

Département de Biologie, Université de Moncton, Moncton, N.-B., E1A $3 E 9$ Canada.

(*) Pêches et Océans, Gouvernement du Canada Halifax, N.-E., B3J 2S7, Canada.

Summary. Effect of adding amino acids to a crab protein diet for juvenile lobsters (Homarus americanus).

Diets containing $30 \%$ crab protein Cancer irroratus (rock crab) were supplemented with one or a combination of the essential amino acids : methionine, histidine and lysine. These diets were fed to juvenile lobsters for 10 weeks. Diets supplemented with lysine and histidine, lysine and methionine or histidine and methionine generally produced greater weight gain and higher survival than did other combinations. These advantages become even more apparent when weight and survival are expressed in terms of Normalized Biomass Increase (NBI). However, none of the test diets produced differences in the duration of molt time, body moisture or mineral content.

\section{Introduction.}

Plusieurs études ont déjà été effectuées dans le but de trouver une source de protéines pouvant satisfaire de façon adéquate les besoins nutritionnels du homard (Castell et Budson, 1974 ; Conklin et al., 1975, 1977 ; Gallagher et al., 1976 ; Boghen et Castell, 1981 ; Lucien-Brun et al., 1985). Par exemple, Castell et Budson (1974) ont démontré que les protéines représentent un besoin nutritionnel important pour les homards allant de 40 à $60 \%$ du poids sec du régime, lorsque la caséine est la seule source protéique utilisée. Conklin et al. (1975) ont rapporté que la caséine et le soja donnent de meilleurs résultats de croissance et de survie que les protéines d'œuf entier ou de chair de poisson. Plus récemment, Boghen et al. (1982) ont trouvé que des protéines extraites de la chair de crabe permettaient de meilleurs taux de croissance et de survie que d'autres sources protéiques telles que la caséine et les protéines provenant de crevettes, moules et oursins. Partant de l'hypothèse que la chair de crabe représente une bonne source de protéines pour les homards juvéniles, il nous a semblé intéressant d'étudier la possibilité d'améliorer ses qualités nutritionnelles en ajoutant certains acides aminés pour augmenter sa valeur. A l'aide de radio-isotopes, on a pu déterminer qualitative- 
ment quels acides aminés sont essentiels pour quelques crustacés décapodes. On a trouvé que le homard a un besoin essentiel pour l'arginine, I'histidine, l'isoleucine, la leucine, la lysine, la méthionine, la phénylalanine, la thréonine, le tryptophane et la valine (Gallagher, 1976). Le crabe semble avoir les mêmes besoins en acides aminés (Lasser et Allen, 1976). Il est possible de réduire la concentration protéique d'un régime si la protéine consommée est nutritionnellement adéquate (Boghen et Castell, 1981 ; Lucien-Brun et al., 1985). Partant de ces principes, nous avons choisi de nourrir des homards juvéniles d'un extrait protéique provenant de chair de crabe et cela à un niveau représentant $30 \%$ du poids sec du régime utilisé. Selon Guthneck (1953) et March et al. (1966), la lysine de diverses protéines alimentaires $n^{\prime}$ est pas toujours complètement disponible au cours du processus de digestion. II semble en être de même pour la méthionine et l'histidine (Castell et Boghen, 1979). Dans la présente étude, nous avons donc choisi de supplémenter l'extrait protéique de chair de crabe avec la lysine, la méthionine et l'histidine, soit isolément soit en combinaison.

\section{Matériel et méthodes.}

Animaux expérimentaux. - Les larves du homard, provenant tous de la même femelle, ont été nourries d'artémies vivantes (Artemia salina) jusqu'au quatrième stade, conformément au procédé décrit par Castell (1977) et, par la suite, d'artémies congelées jusqu'à ce qu'elles parviennent au cinquième stade. Le système d'aquaculture utilisé fut celui décrit par Boghen et Castell (1979) dans lequel les animaux sont maintenus dans de l'eau de mer artificielle (Instant Ocean, Aquarium Systems, Inc.). La disposition des animaux dans des bassins fut inversée au début de chaque semaine afin d'éliminer l'effet de leur emplacement à l'intérieur du système. La qualité de l'eau fut vérifiée soigneusement et des ajustements de température $\left(20^{\circ} \mathrm{C}+2{ }^{\circ} \mathrm{C}\right)$ et de salinité $32 \%+2 \%$ furent effectués afin de maintenir des conditions stables d'environnement. Les homards juvéniles ont été divisés en 8 groupes de 34 individus, lesquels ont été nourris ad libitum deux fois par jour durant 10 semaines ou la durée de l'essai. La nourriture non consommée a été enlevée à l'aide d'un siphon avant chaque repas. Les mues, ainsi que les mortalités, furent notées. Les animaux ont été pesés individuellement toutes les deux semaines (balance Mettler P160 + $1 \mathrm{mg}$ ) après avoir été essorés quelques secondes sur une serviette de papier pour éliminer l'excès d'eau.

Composition et préparation des régimes expérimentaux. - Les ingrédients utilisés ont été ceux de la formule décrite par Boghen et Castell (1981), sauf que la nourriture utilisée dans la présente étude a été congelée à $-70^{\circ} \mathrm{C}$, puis lypholisée durant $24 \mathrm{~h}$. Huit différents suppléments furent étudiés. La composition de ces régimes en acides aminés, méthionine, histidine et lysine, est présentée au tableau 1. Le régime 1, caractérisé par l'ajout de 3,0\% d'acide glutamique, sert de régime témoin. Pour les régimes 2 à 8 , les acides aminés ont été également ajoutés au taux de $3 \%$ du poids sec. La chair de crabe utilisée avait été congelée depuis 4 ans par Tidewater Inc. (Victoria, I.P.E. Canada). Elle a été fournie par le National Sea Products Inc. Cette chair de crabe a été traitée à l'isopropanol chaud 
selon la méthode de Power (1962) pour obtenir le concentré de protéines utilisé comme source protéique des régimes 1 à 8 . Pour formuler chaque régime, les ingrédients secs furent bien mélangés, puis additionnés de petites quantités d'eau distillée à $100^{\circ} \mathrm{C}$, jusqu'à obtention d'une pâte élastique et uniforme. Cette dernière fut mise à solidifier pendant une heure dans un réfrigérateur puis passée au hachoir à viande et mise sous forme de spaghettis de $2 \mathrm{~mm}$ de diamètre, lesquels furent congelés puis lyophilisés. La préparation des régimes, en lots de $100 \mathrm{~g}$, fut faite une semaine avant le début de l'essai nutritionnel. Les régimes lyophilisés furent entreposés dans un incubateur à température constante $\left(10^{\circ} \mathrm{C}\right)$ pendant toute la durée de l'expérience.

\section{TABLEAU 1}

Composition élémentaire des régimes et supplémentation en acides aminés.

\begin{tabular}{lccccccccc}
\hline \multirow{2}{*}{ Ingrédients du régime } & \multicolumn{8}{c}{ \% en poids sec des ingrédients } \\
\cline { 2 - 9 } & 1 & 2 & 3 & 4 & 5 & 6 & 7 & 8 \\
\hline Protéines de crabe & 30,0 & 30,0 & 30,0 & 30,0 & 30,0 & 30,0 & 30,0 & 30,0 \\
L-Glytamine & 3,0 & - & - & - & - & - & - & - \\
DL-Méthionine & - & 3,0 & - & - & 1,5 & - & 1,5 & 1,0 \\
L-Histidine & - & - & 3,0 & - & 1,5 & 1,5 & - & 1,0 \\
L-Lysine monohydrochlorure & - & - & - & 3,0 & - & 1,5 & 1,5 & 1,0 \\
Autres ingrédients $\left({ }^{a}\right)$ & 67,0 & 67,0 & 67,0 & 67,0 & 67,0 & 67,0 & 67,0 & 67,0 \\
\hline
\end{tabular}

(a) Les autres ingrédients utilisés furent : Gélatine 10,0\% ; Carboxyméthylcellulose 2,0\% ; Cellulose 24,3\% ; Amidon de maïs 5,0\% ; Minéraux 5,0\% (Mélange de sels Bernhart-Tomarelli) ; Cholestérol $1,0 \%$, Lécithine 7,5\%; Huile de foie de morue $9,0 \% ;$ Chlorure de choline 1,0\%; Vitamine E, $0,2 \%$; Mélange de vitamines $2,08 \%$.

La composition du mélange de vitamines était la suivante: Thiamine $0,320 \%$; Riboflavine

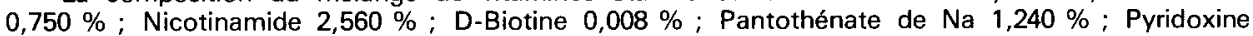
$0,240 \%$; Acide folique $0,096 \%$; Ménadione $0,08 \%$; Cobalamine 0,267 \% ; i-Inositol $12,500 \%$; Acide ascorbique $6,000 \%$; Acide para-amino-benzoïque 2,000\%; Calciférol $0,024 \%$; Hydroxyanisol butylé $0,075 \%$; Hydroxyloluène butylé $0,075 \%$; Acétate de vitamine A $0,500 \%$.

Tous les ingrédients ont été obtenus de ICN Nutritional Biochemicals, Cleveland, Ohio, sauf les vitamines $E, A$ et $D_{3}$, qui proviennent de Roche Chemical Division, Nutley, N. J. et le cholestérol qui provient de Sigma Chemicals, St-Louis, Missouri, USA.

La teneur en eau des chairs de crabe et de homard a été déterminée en séchant les échantillóns dans une étuve à air forcé à $110^{\circ} \mathrm{C}$ durant $24 \mathrm{~h}$, puis en mesurant le poids résiduel. La teneur en minéraux fut obtenue en incinérant la matière sèche au four (Thermolyne) à $550{ }^{\circ} \mathrm{C}$. La teneur en protéines ainsi que l'analyse des acides aminés a été déterminée à l'aide d'un analyseur Kjeldhal automatique (Technicon Auto Analyser II).

L'analyse statistique des données de gains de poids et des valeurs moyennes des $A B N\left({ }^{1}\right)$ a été effectuée à l'aide du test Student-Newman-Keuls (Sokal et Rohlf, 1969). Un test du Chi-carré à un niveau de confiance de $95 \%$ a été utilisé pour établir les différences significatives dans la survie des homards alimentés avec les divers régimes étudiés.

(1) $A B N$ : Explication dans la légende de la figure 1. 


\section{Résultats et discussion.}

Les effets des divers régimes supplémentés sur le gain de poids et sur la survie des homards sont résumés au tableau 2. Les gains de poids moyens s'étendent de $157 \%$ pour le régime $1(\mathrm{C}+\mathrm{G})$ à $235 \%$ pour le régime $4(\mathrm{C}+\mathrm{L})$. Les taux de survie varient de $41 \%$ pour le régime $2(\mathrm{C}+\mathrm{M})$ à $79 \%$ pour le régime 8 $(\mathrm{C}+\mathrm{MHL})$. Les gains de poids moyens des homards juvéniles sont sensiblement les mêmes pendant les quatre premières semaines de l'essai nutritionnel ; des différences plus marquées apparaissent cependant au cours des six semaines suivantes. Le tableau 2 indique que les homards nourris aux régimes supplémentés avec les acides aminés méthionine, histidine et lysine (seuls ou en combinaison) tendent à obtenir un meilleur taux de croissance que ceux nourris avec l'aliment témoin, ce qui témoigne de l'efficacité de la supplémentation. Même si les différences ne s'avèrent pas significatives $(P<0.01)$, le fait que les régimes 4 $(C+L) ; 6(C+H L), 7(C+M L)$ et $8(C+M H L)$ tendent à donner les meilleurs gains de poids, indique également que l'addition de lysine contribue à une stimulation de croissance des homards juvéniles.

TABLEAU 2

Croissance et survie des homards juvéniles nourris avec les régimes artificie/s (supplémentés d'acides aminés) après 10 semaines.

\begin{tabular}{lcccc}
\hline $\begin{array}{c}\text { Composition } \\
\text { protéique } \\
\text { du régime (1) }\end{array}$ & $\begin{array}{c}\text { Poids corporel } \\
\text { initial (mg) }\end{array}$ & $\begin{array}{c}\text { Poids corporel } \\
\text { final }(\mathrm{mg})\end{array}$ & $\begin{array}{c}\text { Gain } \\
\text { de poids moyen }\left(^{2}\right) \\
\%\end{array}$ & $\begin{array}{c}\text { Taux } \\
\text { de survie } \\
\%\end{array}$ \\
\hline $1 \mathrm{C}+\mathrm{G}$ & $98 \pm 35$ & $206 \pm 55$ & $157^{\mathrm{C}}$ & $59^{\mathrm{ABC}}$ \\
$2 \mathrm{C}+\mathrm{M}$ & $91 \pm 33$ & $225 \pm 56$ & $193^{\mathrm{ABC}}$ & $41^{\mathrm{C}}$ \\
$3 \mathrm{C}+\mathrm{H}$ & $90 \pm 39$ & $246 \pm 77$ & $201^{\mathrm{ABC}}$ & $68^{\mathrm{AB}}$ \\
$4 \mathrm{C}+\mathrm{L}$ & $91 \pm 36$ & $286 \pm 74$ & $235^{\mathrm{A}}$ & $53^{\mathrm{BC}}$ \\
$5 \mathrm{C}+\mathrm{MH}$ & $91 \pm 31$ & $237 \pm 53$ & $172^{\mathrm{BC}}$ & $62^{\mathrm{ABC}}$ \\
$6 \mathrm{C}+\mathrm{HL}$ & $91 \pm 33$ & $266 \pm 79$ & $205^{\mathrm{AB}}$ & $74^{\mathrm{A}}$ \\
$7 \mathrm{C}+\mathrm{ML}$ & $86 \pm 39$ & $242 \pm 68$ & $216^{\mathrm{AB}}$ & $74^{\mathrm{A}}$ \\
$8 \mathrm{C} / \mathrm{MHL}$ & $87 \pm 28$ & $256 \pm 78$ & $203^{\mathrm{AB}}$ & $79^{\mathrm{A}}$ \\
\hline
\end{tabular}

$\left({ }^{1}\right)_{C}=$ Crabe, $\mathrm{G}=$ Glutamine, $\mathrm{H}=$ Histidine, $\mathrm{L}=$ Lysine, $\mathrm{M}=$ Méthionine.

$\left({ }^{2}\right)$ Les nombres affectés d'une même lettre ne diffèrent pas significativement $(p<0,05)$.

En observant le tableau 2, on remarque cependant que le régime 4 , composé de protéine de crabe et de lysine, est l'un des régimes donnant le plus faible taux de survie, soit $53 \%$. Ceci est en contraste frappant avec les taux de survie de $74 \%$ à $79 \%$ qui furent obtenus avec les régimes 6,7 et 8 contenant des combinaisons de lysine/méthionine, lysine/histidine ou lysine/méthionine/histidine.

Si l'on compare la composition en acides aminés de la chair de crabes (tabl. 3) a celle des homards (Mason et Castell, 1980) on peut conclure que la lysine n'est pas en elle-même limitante. Au contraire, il est possible que l'addition de $3,0 \%$ de lysine puisse entraîner une déficience relative des autres acides aminés du régime. Cependant, lorsqu'on ajoute $1,5 \%$ de méthionine ou 1,5\% d'histi- 
dine en plus de $1,5 \%$ de lysine, on obtient une augmentation des taux de croissance comparables à ceux du régime 4 mais la survie est nettement améliorée (fig. 1 et tabl. 2). Ces résultats diffèrent de ceux de Mason et Castell (1980) qui

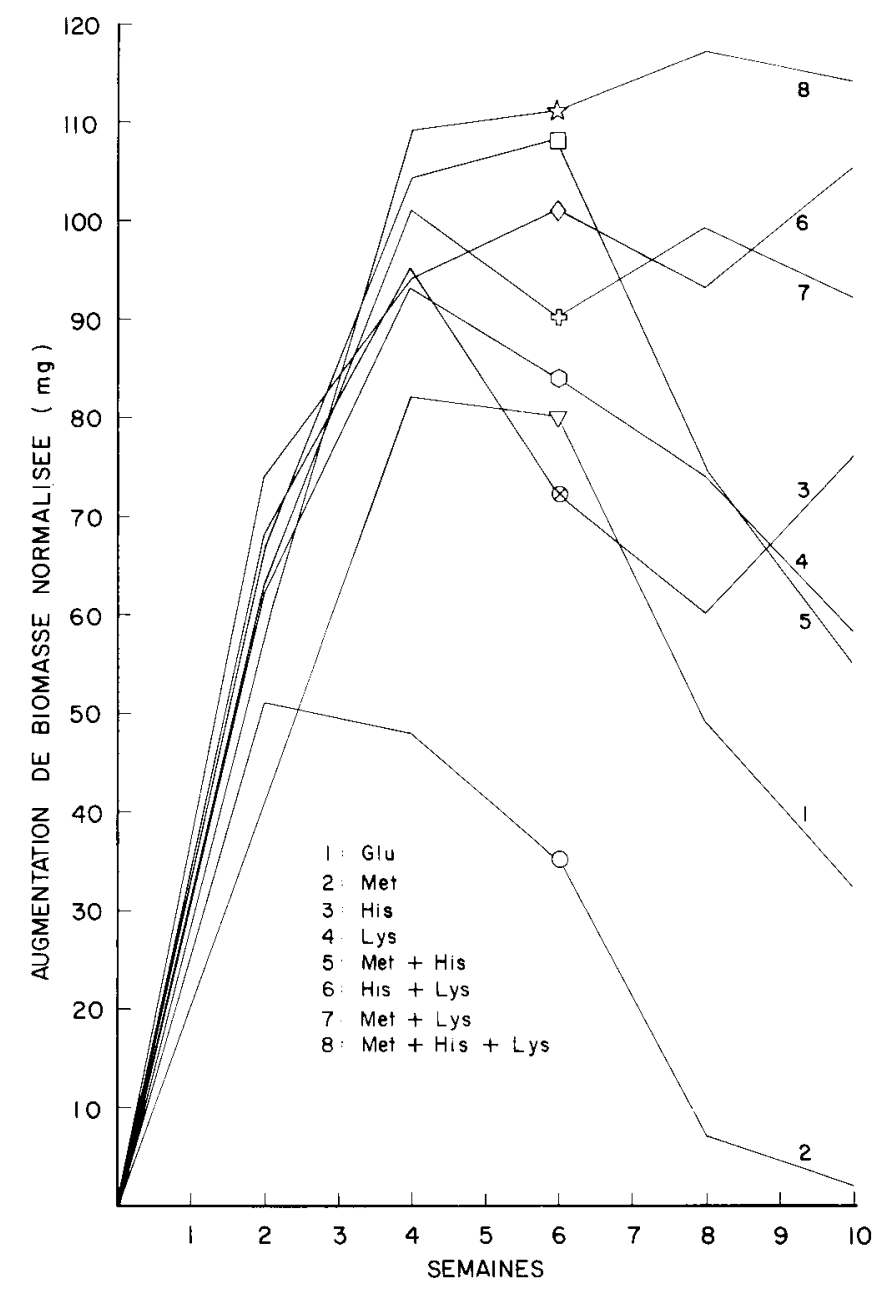

FIG. 1. - Effets des aliments expérimentaux sur l'Augmentation de Biomasse normalisee $(A B N)(a)(b)$.

(a) L'Augmentation de Biomasse Normalisée (Conklin et al., 1975) est une expression qui tient compte à la fois du gain de poids et de la survie des animaux. Elle est déterminée selon la formule :

$\mathrm{ABN}=\mathrm{Pf} \times \mathrm{Nf}-\mathrm{Pi} \times \mathrm{Ni}$ ou $\mathrm{Pf}=$ poids moyen final, $\mathrm{Nf}=$ nombre d'animaux survivants, $\mathrm{Ni}$

$\mathrm{Pi}=$ poids moyen initial, $\mathrm{Ni}=$ nombre initial d'animaux.

(b) Les régimes soulignés par une même ligne ne diffèrent pas significativement :

$\begin{array}{llllllll}C-M & C-G & C-M H & C-L & C-H & C-M L & C-H L & C-M H L\end{array}$ 
ont supplémenté un concentré de protéines de morue avec la lysine en combinaison avec d'autres acides aminés. Dans cette étude, le supplément de lysine semblait retarder la croissance des homards juvéniles ; ces derniers indiquent toutefois qu'une trop grande supplémentation $(3,9 \%)$ de cet acide aminé a pu en masquer les conséquences, à cause des effets nocifs de la lysine à des concentrations trop élevées (Harper et al., 1970). D’ailleurs Martin (1980) a également démontré que l'ajout d'acides aminés purifiés aux régimes de la crevette Palaemon serratus peut produire des effets inhibant la croissance ; cet effet se reflète sur la nature et le taux optimal de l'acide aminé considéré.

II est possible que nos résultats s'expliquent par la " théorie aminostatique » qui indique que des excès ou des déficiences d'acides aminés du sang sont directement responsables pour la prise de nourriture (Mellinkoff et al., 1956) et que la quantité de nourriture mangée se traduit en croissance (Kissileff et Van Italie, 1982).

Le crabe congelé utilisé dans la présente expérience s'est avéré d'une valeur nutritive inférieure au crabe frais utilisé précédemment (Boghen et al., 1982) lequel a permis un gain de poids moyen de $408 \%$ et un taux de survie de $95 \%$ en 11 semaines. Cette infériorité ne peut être attribuée à des différences de composition en acides aminés du crabe frais et du crabe congelé car, comme l'indique le tableau 3, les acides aminés de ces deux sources de protéines sont semblables. D'autres facteurs peuvent possiblement expliquer les résultats inférieurs obtenus avec le crabe congelé : 1) la congélation pourrait entraîner une dénaturation de la protéine réduisant la disponibilité des acides aminés (Finn, 1934 ; Snow, 1950) ;

TABLEAU 3

Composition en acides aminés de chair de crabe congelée et fraîche $(\mathrm{g} / 100 \mathrm{~g}$ protéine).

\begin{tabular}{lcc}
\hline \multicolumn{1}{c}{ Acide aminé } & $\begin{array}{c}\text { Crabe congelé } \\
\text { pendant } 4 \text { ans }\end{array}$ & Crabe frais \\
\hline Alanine & 4,5 & 4,5 \\
Arginine* & 6,1 & 6,9 \\
Acide aspartique & 8,4 & 8,1 \\
Cystéine & - & - \\
Glycine & 3,6 & 3,5 \\
Acide glutaminique & 12,5 & 12,7 \\
Histidine* & 2,1 & 2,0 \\
Isoleucine* & 4,6 & 3,8 \\
Leucine $^{*}$ & 6,9 & 6,4 \\
Lysine $^{*}$ & 7,2 & 6,9 \\
Méthionine* & 2,6 & 2,6 \\
Phénylalanine & 4,3 & 3,5 \\
Proline & 3,3 & 2,9 \\
Sérine & 3,5 & 3,3 \\
Thréonine & 3,8 & 3,6 \\
Tryptophane & - & $-3,2$ \\
Tyrosine & 3,5 & 4,0 \\
Valine $^{*}$ & 4,4 & 77,9 \\
TOTAL & 81,0 & \\
\hline
\end{tabular}

* identifié comme essentiel pour les homards juvéniles (Gallagher, 1976). 
2) la congélation prolongée pourrait réduire le taux de digestibilité des protéines de la chair de crabe pour des homards juvéniles.

L'analyse de la composition corporelle des homards juvéniles a indiqué que leur teneur moyenne en matière sèche était de $21,8 \%$ du poids frais et que leur teneur en matières minérales était de $35,1 \%$ du poids sec. II n'y eut aucune différence statistiquement significative reliée aux régimes expérimentaux. Cela indique que les différences observées dans le gain de poids sont le résultat d'une synthèse réelle de tissus et non de différences dans la rétention de l'eau ou l'assimilation de minéraux.

Les homards juvéniles ont mué en moyenne 2,2 fois au cours des 10 semaines de l'essai nutritionnel. La durée moyenne de la période d'intermue fut de 23,4 jours (stade 5 au stade 6) et de 36,9 jours (stade 6 au stade 7). Aucune différence significative n'a été trouvée dans le nombre et la durée moyenne des périodes d'intermue en fonction des divers régimes.

Les régimes utilisés dans cette étude ont été supplémentés avec divers niveaux d'acides aminés, soit de $1,0 \%$, de $1,5 \%$ ou de 3,0 \%. En supposant que les homards auraient pu utiliser une partie de ces acides aminés comme source d'énergie, les différences en rendement calorique des suppléments d'acides aminés étudiés auraient été négligeables.

Les résultats indiquent que, d'une façon générale, les acides aminés supplémentés en groupes de 2 ou 3 furent plus efficaces que ceux utilisés isolément. Les régimes 2 et 3 supplémentés avec $3 \%$ chacun de méthionine, et d'histidine, supportèrent moins bien la croissance. Le régime 4 supplémenté avec $3 \%$ de lysine donne le meilleur taux de croissance, mais ne permet que $53 \%$ de survie des homards. Par contre, les régimes $5,6,7$ et 8 où deux ou trois acides aminés étaient combinés, chacun contribuant pour 1 ou $1,5 \%$ du poids du régime, donnent les meilleurs résultats. Cela ne nous renseigne toutefois pas sur le rôle spécifique d'un acide aminé particulier dans une telle combinaison. Une étude supplémentaire avec des aliments expérimentaux ayant un même niveau de supplémentation pour chaque acide aminé serait nécessaire pour évaluer le rôle spécifique de chaque acide aminé à l'intérieur d'une combinaison. Il faut également introduire dans la mesure du possible un meilleur contrôle de la quantité totale de nourriture consommée.

Finalement, de telles études doivent être effectuées à différents moments au cours du cycle de vie du homard, car les besoins physiologiques, donc protéiques, changent en fonction de l'âge.

Reçu en février 1985.

Accepté en septembre 1986.

Remerciements. - Ce travail a été réalisé grâce à une subvention octroyée par le Ministère des Pêches et Océans du Canada au Dr. A. Boghen. Les auteurs tiennent à remercier très chaleureusement le Professeur H. J. Ceccaldi et le Professeur A. Cormier pour avoir relu le manuscrit et pour nous avoir fait bénéficier de leurs commentaires. 


\section{References}

BOGHEN A. D., CASTELL J. D., 1979. A recirculating system for small scale experimental work on juvenile lobsters Homarus americanus. Aquaculture, 18, 383-387.

BOGHEN A. D., CASTELL J. D., 1981. Nutritional value of different dietary proteins to juvenile lobster Homarus americanus. Aquaculture, 22, 343-351.

BOGHEN A. D., CASTELL J. D., CONKLIN D., 1982. In search of a reference protein to replace vitamin-free casein in lobster nutrition studies. Can. J. Zool., 60, 2033-2038.

CASTELL J. D., 1977. Production of juvenile lobsters Homarus americanus for nutrition research. Actes Colloques CNEXO, 4, 277-281.

CASTELL J. D., BOGHEN A. D., 1979. Fatty acid metabolism in juvenile lobsters Homarus americanus) fed a diet low in methionine and histidine. Proc. World Maricul. Soc., 10, 720-727.

CASTELL J. D., BUDSON S. D., 1974. Lobster nutrition : The effects on Homarus americanus of dietary protein levels. J. Fish. Res. Bd. Can., 31, 1363-1370.

CONKLIN D. E., DEVERS K., SHLESER R. A., 1975. Initial development of artificial diets for the lobster Homarus americanus. Proc. World Maricul. Soc., 6, 237-248.

CONKLIN D. E., DEVERS K., BORDNER C., 1977. Development of artificial diets for the lobster Homarus americanus. Proc. World Maricul. Soc., 8, 841-852.

FINN D. B., 1934. The denaturation of fish muscle protein by freezing. Contrib. Canad. Biol., 8 (25) 311-320.

GALLAGHER M. L., 1976. The nutritional requirement of juvenile lobsters Homarus americanus. Ph. D. thesis, University of California, Davis, U.S.A.

GALLAGHER M. L., CONKLIN D. E., BROWN W. D., 1976. The effects of pelletized protein diets on growth, molting and survival of juvenile lobsters. Proc. World Maricul. Soc., 7, 363-378.

GUTHNECK B. T., 1953. Utilization of amino acids from food by the rat. II. Lysine. J. Nutr., 49. 289-294.

HARPER A. E., BENEVENGA N. J., WOBLHEUTER R. M., 1970. Effects of ingestion of disproportionate amounts of amino acids. Physiol. Rev., 50, 428-558.

KISSILEFF H. R., VAN ITALLIE T. B., 1982. Physiology of the control of food intake. In DARBY W. J., BROQUIST H. P., OLSON R. E., Ann. Rev. Nutr., 2, 371-418.

LASSER G. W., ALLEN W. A., 1976. The essential amino acid requirement of the Dungeness crab, Cancer magister. Aquaculture, 7, 235-244.

LUCIEN-BRUN H., Van WORMHOUDT A., LACHAUX A., CECCALDI H. J., 1985. Effets de régimes composés sur la croissance de homards juvéniles Homarus gammarus L. Estimation biochimique de la composition optimale du régime alimentaire en protéines. Aquaculture, $\mathbf{4 6 ,}$ 97-109.

MARCH B. E., BIELY J., GOUDIE C., TARR H. L. A., 1966. Protein nutritive quality of commercial fish meals as assessed by biological and chemical methods. J. Fish. Res. Bd. Can., 23, 395-414.

MARTIN B. J., 1980. Croissance de la crevette Palaemon serratus (Crustacea Decapoda) nourrie avec des aliments composés enrichis en arginine, methionine, isoleucine et phénylalanine. Tethys, 9, 261-265.

MELLINKOFF S. M., FRANKLAND M., BOYLE D., GREIPEL M., 1956. Relationship between serum amino acid concentration and fluctuations in appetite. J. appl. Physiol., 8, 535-538.

MASON E. G., CASTELL J. D., 1980. The effects of supplementing purified proteins with limiting essential amino acids on growth and survival of juvenile lobsters (Homarus americanus). Proc. World. Maricul. Soc., 11, 346-354.

POWER H. E., 1962. An improved method for the preparation of fish protein concentrate from cod. J. Fish. Res. Bd. Can., 19, 1039-1045.

SNOW J. M., 1950. Protein in fish muscle. III. Denaturation of myosin by freezing. J. Fish. Res. Bd. Can., 7, 599-607.

SOKAL R. R., ROHLF F. J., 1969. Biometry. W. H. Freeman and Co., San Francisco California. $776 \mathrm{pp}$. 